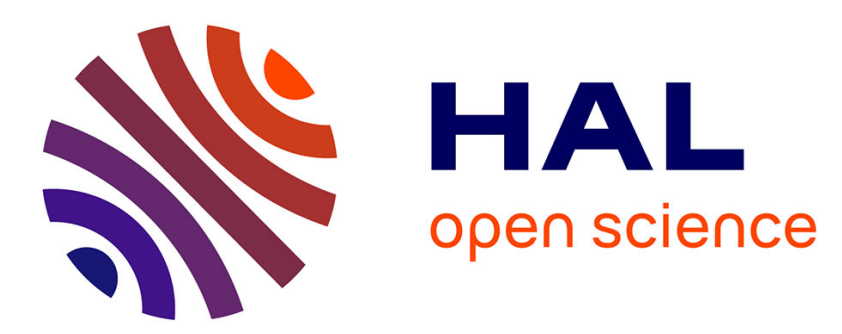

\title{
The effect of the size of the opening on the acoustic power radiated by a reed woodwind instrument
}

Alexis Guilloteau, Philippe Guillemain, Jean Kergomard, Michael Jousserand

\section{To cite this version:}

Alexis Guilloteau, Philippe Guillemain, Jean Kergomard, Michael Jousserand. The effect of the size of the opening on the acoustic power radiated by a reed woodwind instrument. Journal of Sound and Vibration, 2015, 343, pp.166-175. hal-01094511v2

\section{HAL Id: hal-01094511 \\ https://hal.science/hal-01094511v2}

Submitted on 25 Mar 2015

HAL is a multi-disciplinary open access archive for the deposit and dissemination of scientific research documents, whether they are published or not. The documents may come from teaching and research institutions in France or abroad, or from public or private research centers.
L'archive ouverte pluridisciplinaire HAL, est destinée au dépôt et à la diffusion de documents scientifiques de niveau recherche, publiés ou non, émanant des établissements d'enseignement et de recherche français ou étrangers, des laboratoires publics ou privés. 


\title{
The effect of the size of the opening on the acoustic power radiated by a reed woodwind instrument
}

\author{
Alexis Guilloteau ${ }^{a}$, Philippe Guillemain ${ }^{a}$, Jean Kergomard ${ }^{a}$ and Michael \\ Jousserand $^{b}$ \\ ${ }^{a}$ LMA, CNRS, UPR 7051, Aix-Marseille Univ, \\ Centrale Marseille, 13402 Marseille Cedex 20, France \\ ${ }^{b}$ Buffet Group, 5, rue Maurice Berteaux \\ 78711 Mantes-la-Ville, France
}

\begin{abstract}
For a given note, the maker of woodwind instruments can choose between different sizes for the toneholes under the condition that the location is appropriate. The present paper aims at analyzing the consequences of this choice on the power radiated by a hole, which depends on the coupling between the acoustic resonator and the excitation mechanism of the self-sustained oscillation, thus on the blowing pressure. For that purpose a simplified reed instrument is investigated, with a cylindrical pipe and a unique orifice at the pipe termination. The orifice diameter was varied between the pipe diameter and a size such that the instrument did not play. The pipe length was in each case adjusted to keep the resonance frequency constant. A simple analytical model predicts that, for a given mouth pressure of the instrumentalist, the radiated power does not depend on the size of the hole if it is wide enough and if resonator losses are ignored. Numerical solution of a model including losses confirms this result: the difference in radiated power between two diaphragm sizes remains smaller than the difference obtained if the radiated power would be proportional to the orifice cross section area. This is confirmed by experiments using an artificial mouth, but the results show that the linear losses are underestimated, and that significant nonlinear losses occur. The measurements are limited to the acoustic pressure at a given distance of the orifice. Experiments also show that rounding edges of the orifice reduces nonlinear losses resulting in an increase of the power radiated and of the extinction threshold, and resulting in a larger dynamical range.
\end{abstract}


Keywords: Musical acoustics Clarinet-like instruments Woodwinds PACS: $43.75 . \mathrm{Pq}$

\section{Introduction}

When designing a woodwind instrument, for a given note, the maker can choose between a large range of sizes and locations for the toneholes. If the choice is a very wide hole, the effect is close to that of cutting the pipe at the hole location, at least at low frequencies. However it is also possible to choose a narrower hole with a location chosen closer to the pipe inlet than a wider hole, because for a narrow hole the influence of the downstream portion of the main pipe is large.

The present paper aims to investigate whether the above mentioned choice is important for the amplitude of the radiated acoustic power. Obviously if the hole has a vanishing size, the acoustic power radiated by this hole approaches zero. But what happens when the size of the hole increases? To our knowledge, this question has not yet been treated in the literature. Models and measurements for toneholes and tonehole radiation can be found in several papers (see, e.g., $[1,2,3,4])$. However the power radiated by wind instruments in functioning, i.e., including the effect of the nonlinear coupling with an excitor, has rarely been treated in the literature (see [5]). Preliminary results of our study were described in a conference paper [6].

The answer to the above question is are necessarily intuitive, because at low frequencies the real part of the radiation acoustic impedance (which is defined as the ratio of acoustic pressure to acoustic flow) does not depend on the size of the hole (see e.g. [7]).

In the present paper we consider a simplified reed instrument: a cylindrical pipe terminated in only an orifice, and excited by a clarinet-like reed (with mouthpiece). The dependency of the radiated acoustic power on the blowing pressure is investigated. The effect of this simplification is discussed in Section 2.4. The scope of this paper is limited to the case of the notes corresponding to the first register, i.e., when the playing frequency is close to that of the first impedance peak.

The geometry is shown in Fig. 1. The radius of the pipe corresponds to the usual value of the output of a clarinet mouthpiece, $a=7.45 \mathrm{~mm}$. The termination of the pipe is a cylindrical diaphragm of length $\ell_{d}=5 \mathrm{~mm}$, which is approximately equal to the wall thickness of a clarinet, and of radius $b$, 


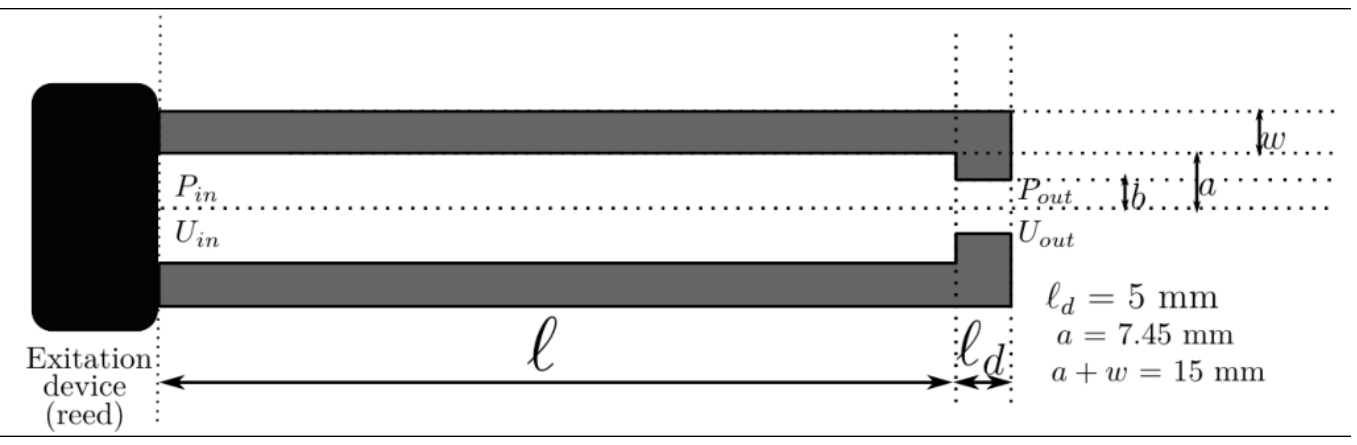

Figure 1: Geometry of the tube with diaphragm. The length $\ell$ depends on the diaphragm radius $b$, with a fixed first resonance frequency $f_{1}=250 \mathrm{~Hz}$.

which is chosen among the following values: $b=7.45,6,5,4,3,2 \mathrm{~mm}$ (The first value corresponds to $b=a$, a pipe without diaphragm). For a clarinet, the tonehole radius varies from $2.5 \mathrm{~mm}$ in the higher part of the instrument to $6 \mathrm{~mm}$ for the hole which is close to the bell. The length $\ell$ of the pipe is chosen in order to keep the first resonance frequency independent of the diaphragm radius and equal to $250 \mathrm{~Hz}$. Thus $\ell$ depends on the diaphragm radius. Without diaphragm, if the sound velocity in free space at $20^{\circ} \mathrm{C}$ is $c=343.4 \mathrm{~ms}^{-1}, \ell$ is equal to $328 \mathrm{~mm}$. With a diaphragm, the length $\ell$ is equal to $323,317,306,286,237 \mathrm{~mm}$ for the widest to the narrowest radius, respectively (see Section 2.2 ). The pipe wall thickness is $w=7.55 \mathrm{~mm}$ (thus the external pipe radius is $a+w=15 \mathrm{~mm}$ ).

In Section 2, a simplified model is proposed. Because losses are ignored, and the pressure signal is assumed to be mostly monochromatic, a simple result is obtained: under certain conditions imposed on the hole radius $b$, the radiated power is independent of this radius. In Section 3, losses are introduced, resulting in a slight modification of the simple result. Then in Section 4, numerical simulation of the sound production and radiation is carried out with an ab initio model, in order to obtain better precision. Finally experiments are presented in Section 5 and compared to the theoretical results.

\section{Elementary theoretical analysis}

\subsection{Simplified linear model of the resonator}

For the calculation of the radiated power, two transfer functions of the resonator have to be determined in the frequency domain: the transfer ad- 
mittance between the output flow rate $U_{\text {out }}$ and the input pressure $P_{\text {in }}$ (in the mouthpiece) for the radiation, and the input impedance $Z_{\text {in }}=P_{\text {in }} / U_{\text {in }}$ for the coupling with the excitation mechanism. For these transfer functions, the simplest model of the present section is based on standard formulas. It ignores the resonator losses. Assuming, however, that at low frequencies $k b<<1$, ( $k$ is the wave number) and therefore that the diaphragm radiates into infinite space as a monopole, the mean radiation power can be deduced from the knowledge of the flow rate $U_{\text {out }}$, calculated when ignoring losses:

$$
\mathcal{P}_{r}=\frac{1}{2} \operatorname{Re}\left(Z_{r}\right)\left|U_{\text {out }}\right|^{2}
$$

where $Z_{r}$ is the radiation impedance of a monopole:

$$
\operatorname{Re}\left(Z_{r}\right)=\frac{k^{2} \rho c}{4 \pi} .
$$

$c$ is the speed of sound and $\rho$ is the air density. The cross-section areas of the pipe and diaphragm are denoted $S=\pi a^{2}$ and $S_{d}=\pi b^{2}$, respectively. The model is based on the following transfer matrix relationship:

$$
\begin{aligned}
& \left(\begin{array}{c}
P_{\text {in }} \\
U_{\text {in }}
\end{array}\right)=\mathbf{T}\left(\begin{array}{cc}
1 & \mathrm{j} \omega\left(M_{d+} M_{\text {disc }}\right) \\
0 & 1
\end{array}\right)\left(\begin{array}{c}
P_{\text {out }} \\
U_{\text {out }}
\end{array}\right) \\
& \text { where } \mathbf{T}=\left(\begin{array}{cc}
\cos (k \ell) & \mathrm{j} Z_{c} \sin (k \ell) \\
\mathrm{j} Z_{c}^{-1} \sin (k \ell) & \cos (k \ell)
\end{array}\right) .
\end{aligned}
$$

$\omega$ is the angular frequency, $Z_{c}=\rho c / S$ is the characteristic impedance, and $\mathrm{j}^{2}=-1$. The compressibility effect inside the diaphragm is ignored; this implies $k \ell_{d}<<1$. The values of the acoustic masses $M_{d,}, M_{d i s c}$ are discussed in the Appendix, as well as the radiation contribution, which is that of an acoustic mass $M_{r}$ : $P_{\text {out }}=\mathrm{j} \omega M_{r} U_{\text {out }}$ (no radiation losses are considered). Therefore it can be checked that Eq. (3) implies:

$$
\left(\begin{array}{c}
P_{\text {in }} \\
U_{\text {in }}
\end{array}\right)=\mathbf{T}\left(\begin{array}{cc}
1 & \mathrm{j} \omega M_{t} \\
0 & 1
\end{array}\right)\left(\begin{array}{c}
0 \\
U_{\text {out }}
\end{array}\right)
$$

where $M_{t}=M_{d}+M_{d i s c}+M_{r}$. In this low-frequency analysis, $\omega M_{t} / Z_{c}$ increases when $b$ decreases. 


\subsection{Input impedance and transfer admittance of the resonator}

If both visco-thermal and radiation losses are ignored, the input impedance can be deduced from Eq. (5). Let us define a length equivalent to the diaphragm as:

$$
k \ell_{e q} \stackrel{\text { def }}{=} \arctan \left[\frac{\omega M_{t}}{Z_{c}}\right] .
$$

Eq. (5) can be rewritten as:

$$
\left(\begin{array}{c}
P_{\text {in }} \\
U_{\text {in }}
\end{array}\right)=\left(\begin{array}{cc}
\cos (k \ell) & \mathrm{j} Z_{c} \sin \left[k\left(\ell+\ell_{\text {eq }}\right)\right] / \cos \left(k \ell_{\text {eq }}\right) \\
\mathrm{j} Z_{c}^{-1} \sin (k \ell) & \cos \left[k\left(\ell+\ell_{\text {eq }}\right)\right] / \cos \left(k \ell_{\text {eq }}\right)
\end{array}\right)\left(\begin{array}{c}
0 \\
U_{\text {out }}
\end{array}\right) .
$$

Therefore the input impedance and the transfer admittance are found to be:

$$
\begin{aligned}
Z_{\text {in }} & =\mathrm{j} Z_{c} \tan \left[k\left(\ell+\ell_{e q}\right)\right] ; \\
\frac{U_{\text {out }}}{P_{\text {in }}} & =\frac{-\mathrm{j}}{Z_{c}} \frac{\cos \left(k \ell_{e q}\right)}{\sin \left[k\left(\ell+\ell_{e q}\right)\right]} .
\end{aligned}
$$

The equivalent length $\ell_{e q}$ is frequency dependent: it decreases when the frequency increases, therefore the diaphragms generate positive inharmonicity for the resonance frequencies of the pipe ("positive" means that the resonance frequencies above the first resonance frequency are higher than exact multiple of it). For the case without diaphragm $b=a$, the choice of the total length $\ell+\ell_{d}$ implies that the first resonance frequency is given by $f_{1}=c / 4 /\left(\ell+\ell_{e q}\right)$, where $\ell_{e q}=\ell_{d}+\delta_{d} a\left(\delta_{d} \simeq 0.7\right.$, see the Appendix). For the other cases the same resonance frequency is chosen, then the equivalent length $\ell_{e q}$ is given by Eq. (6) and the length $\ell$ is deduced from Eq. (8), with an infinitely large input impedance $Z_{\text {in }}$ :

$$
\ell=\frac{\pi}{2 k_{1}}-\ell_{e q}
$$

$\left(k_{1}=\omega_{1} / c\right.$ with $\left.\omega_{1}=2 \pi f_{1}\right)$. At lower frequencies, the transfer admittance simplifies to the constant value $\left(-\mathrm{j} / Z_{c}\right)$. This is valid if the following condition is fulfilled:

$$
k_{1} \ell_{e q}<<1 \text { or } \omega_{1} M_{t}<<Z_{c} .
$$

It follows that if the diaphragm is not too narrow, Eq. (2) implies that the diaphragm radius has no effect on the radiated power for a given input pressure $P_{i n}$. For instance, if

$$
k_{1} \ell_{e q}<0.3
$$


the discrepancy induced by the approximation of the cosine function in Eq. (9) is less than $0.4 d B$. Furthermore the radius should be larger than $3 \mathrm{~mm}$. It can be shown that these conditions are satisfied by most toneholes of a clarinet.

\subsection{Reed and mouthpiece coupled to the resonator}

In order to analyze the relationship between the radiated power and the instrumentalist mouth pressure $p_{m}$, it is necessary to take into account the excitation mechanism. For this purpose, the model proposed by Wilson and Beavers [8] is acceptable. It can be simplified by ignoring the reed dynamics. This results in a quasi-static nonlinear characteristic $u_{i n}=F\left(p_{i n}\right)$, where $p_{i n}(t)$ and $u_{i n}(t)$ are the inverse Fourier transform of $P_{i n}(\omega)$ and $U_{i n}(\omega)$, respectively:

$$
u_{\text {in }}=F\left(p_{i n}\right)=u_{A}\left[1-\Delta p / p_{M}\right] \sqrt{|\Delta p| / p_{M}} \operatorname{sign}(\Delta p) .
$$

$p_{M}$ is the closure pressure, proportional to the reed stiffness, $\Delta p=p_{m}-p_{i n}$, where $p_{m}$ is the mouth pressure, assumed to be constant, $u_{A}$ is proportional to the maximum flow rate entering the instrument (see $[8,9]$ ). The analytical model used here considers the case of negative flow rate, whose possible occurrence should be taken into account within the digital simulations that use a truncated modal series of the impedance. However for clarinet-like instrument, a negative flow rate is usually not encountered (see Ref. [10]). The simplest control parameters that can be defined with such a model are the mouth pressure $p_{m}$ and the reed channel opening area $S_{c}$ at rest. Using dimensionless quantities, $\widetilde{p}_{i n}=p_{i n} / p_{M}$ and $\widetilde{u}_{i n}=u_{i n} Z_{c} / p_{M}$, Eq. (13) can be written in dimensionless quantities:

$$
\widetilde{u}_{i n}=\zeta\left(1-\gamma+\widetilde{p}_{i n}\right) \sqrt{\gamma-\widetilde{p}_{i n}},
$$

where $\gamma=p_{m} / p_{M}$ and $\zeta=Z_{c} S_{c} \sqrt{2 /\left(\rho p_{M}\right)}$, (see [8]). These equations hold for a non-beating reed (this implies approximately $\gamma<1 / 2$ ), and a Taylor expansion can be derived for Eq. (14) around $p_{\text {in }}=0$. For the following, we need the values of the polynomial coefficients up to order 3 [11]:

$$
A=\zeta \frac{3 \gamma-1}{2 \sqrt{\gamma}} ; B=-\frac{\zeta(3 \gamma+1)}{8 \gamma^{3 / 2}} ; C=-\zeta \frac{\gamma+1}{16 \gamma^{5 / 2}}
$$

When no diaphragm is present the resulting pressure signal $p_{i n}(t)$ is a square signal, because resonator losses are ignored. Then it is easily shown 
that assuming the validity of Eq.(2) the radiated pressure is the derivative of a square signal. At this point a problem occurs: a signal such as the one discussed above would correspond to an infinite power. The reason is that Eq.(2) is not valid at higher frequencies. The model without losses corresponds to the degenerate case studied in Ref. [12]. For an elementary analysis we use the approximation of the first harmonic, which approximate the square wave function by its first Fourier component. We will refine the calculation in Section 4. This difficulty is probably not encountered with diaphragms, because inharmonicity occurs (see Eq.(6)) and the fundamental frequency is dominant, but the comparison with the tube without diaphragm would be meaningless. The following relationship has been found for the first Fourier component $[12,11]$ :

$$
P_{i n}\left(\omega_{1}\right)=p_{M} \sqrt{\frac{Y_{1}-A}{3 C}}
$$

$Y_{n}=Z_{c} / Z_{i n}\left(n \omega_{1}\right)$ is the dimensionless input admittance. Eq. (16) implies that $Y_{1}$ is real [12], and therefore the value of the operating frequency can be calculated. Because the resonator losses are ignored, $Y_{1}=0$, thus the playing frequency is $f_{1}$ and the input pressure $P_{i n}\left(\omega_{1}\right)$ at frequency $f_{1}$ does not depend on the radius of the diaphragm; this is also true for the output flow rate $U_{\text {out }}\left(\omega_{1}\right)$, as well as for the radiated power. To summarize the assumptions of this result: the diaphragm is wide enough (condition (11)); no resonator losses are considered; and the input acoustic pressure is reduced to a quasi-sinusoidal signal. The value of the power is therefore:

$$
\mathcal{P}_{r}=\frac{k_{1}^{2} \rho c}{8 \pi} \frac{1}{Z_{c}^{2}} p_{M}^{2}\left|\frac{-A}{3 C}\right| .
$$

Notice that when losses are ignored the oscillation threshold $\gamma=1 / 3(A=0)$ is also independent of the diaphragm opening.

\subsection{Generalization to a side tonehole}

It is possible to generalize this elementary approach to the case of a unique side hole, with some restrictive hypotheses, since it is impossible to treat the general case of a complete lattice of toneholes in a simple way. Obviously, the difference in flow can be important, but it is ignored in this discussion, which remains very elementary. Consider a pipe of total length $L$, and one tonehole located at distances $\ell_{\text {down }}$ and $\ell$ from the pipe output and input, 
respectively. When it is closed, the frequency is $c /(4 L)$, and when it is open, it is $c /\left(4 L_{1}\right)$, with $L=\ell+\ell_{\text {down }}$ and $L_{1}=\ell+\ell_{e q}$. We suppose here that there is one semi-tone between the two frequencies, i.e., $L_{1} \simeq 0.94 \mathrm{~L}$. At low frequencies, the pipe portion downstream the hole can be considered as an acoustic mass, $\rho \ell_{\text {down }} / S$, in parallel with the acoustic mass of the hole $M_{h}$ and that of the pipe. Consequently we can use the model given by Eq. (3), replacing the mass $M_{t}$ by $M_{d h}$ given by:

$$
\frac{1}{M_{d h}}=\frac{1}{M_{h}}+\frac{S}{\rho \ell_{\text {down }}}
$$

In order to use the same analysis as is used for a diaphragm, $M_{d h}$ needs to be small; more specifically $M_{d h}^{-1}>M_{h}^{-1}$, therefore if the acoustic mass of the hole is sufficiently small, the analysis applies. Moreover the flow rate $U_{\text {out }}$ entering the mass $M_{d h}$ is the sum of the flow rates going out of the hole and of the pipe. If the distance $\ell_{\text {down }}$ is smaller than a wavelength, the total flow rate radiates as a single monopole in the far field, and Eq. (1) remains valid. Therefore the elementary analysis remains valid if the hole is wide enough.

\section{Improved analytical model (non-beating reed)}

The above analysis remains largely qualitative. In order to improve the model, visco-thermal effects (losses and dispersion) are considered by using the standard formula of transmission lines for both the pipe and the diaphragm. The wavenumber $k$ and characteristic impedances are calculated at the second order of the Stokes number [13]. The computation of the input impedance shows that: i) the peaks of the input impedance decrease when the diaphragm radius decreases: this means that the losses inside the diaphragm increase and compensate for the diminution of the losses inside the pipe due to the length decrease (for the two extreme cases of $b=a$ and $b=2 \mathrm{~mm}$, the difference is $3.5 \mathrm{~dB}$ ); ii) inharmonicity between the two first peaks increases when the diaphragm radius decreases, as noticed in Section 2.2 .

The effect of the losses is a diminution of the power for the first harmonic, due to the decrease of the numerator in Eq. (17), the coefficient $A$ being replaced by $A-Y_{1}$. Losses increase when the radius decreases, and, because $\operatorname{Re}\left[Y_{1}\right]$ is positive, the oscillation threshold also increases (see Eq.(16)): it is the reason why the radiated power is strongly modified near the oscillation threshold. 


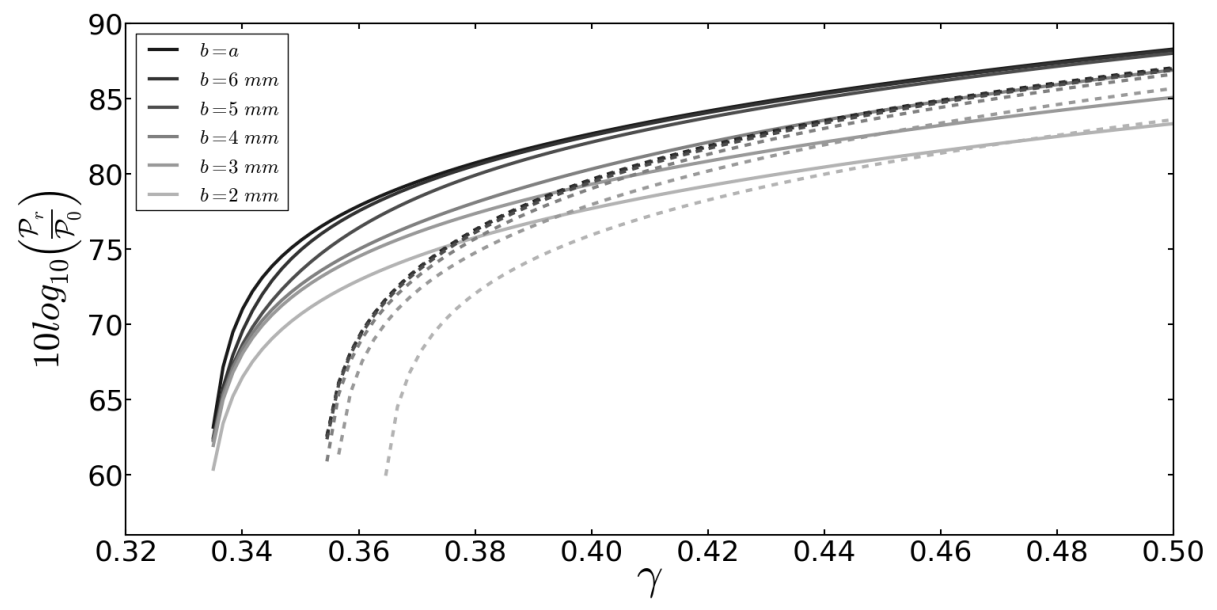

Figure 2: Calculated results from analytical formulas (16) and (19), for the 3 first harmonics below the beating reed regime $(\gamma=1 / 2)$. Dashed and plain lines correspond to the calculation with and without losses, respectively. The line shading is proportional to the diaphragm radius. Notice that losses produce both a shift of threshold and a decrease of the power.

The calculation is limited to the sum of the 3 first harmonics and to the range $\gamma<1 / 2$; it is done for $\zeta=0.43$, a reasonable value for clarinet mouthpiece and reed. The calculation of the amplitude of the harmonics 2 and 3 have been done by using the results of the variable truncation method [11]:

$$
\frac{P_{3}}{P_{1}} \simeq-\frac{1}{3} \frac{A-Y_{1}}{A-Y_{1}+Y_{3}-Y_{1}} \text { and } \frac{P_{2}}{P_{1}} \simeq \frac{B\left(P_{1}^{2}+2 P_{3} P_{1}\right)}{Y_{2}} .
$$

The results for the total power of the 3 harmonics are shown in Fig. 2, and compared to the results of the simplest approximation (a single Fourier component) when losses are ignored. The value of the radiated power, reduced by the reference power $\mathcal{P}_{0}=10^{-12} \mathrm{~W} \cdot \mathrm{m}^{-2}$, is drawn with respect to the dimensionless excitation pressure $\gamma$. Different values of diaphragm radius, with and without losses, are considered, and the closure pressure is $p_{M}=5 \mathrm{kPa}$ (see Eq. (17)). It appears that between the radii $b=5 \mathrm{~mm}$ and $b=a$ the difference is less than $1 \mathrm{~dB}$ above $\gamma=0.4$, while if the power ratio were proportional to the hole area, the difference would be $3.5 \mathrm{~dB}$. Between the two extreme cases, the difference is $4 d B$ instead of $11.5 \mathrm{~dB}$.

Notice that the $2 n d$ and $3 r d$ harmonics do not increase drastically the 


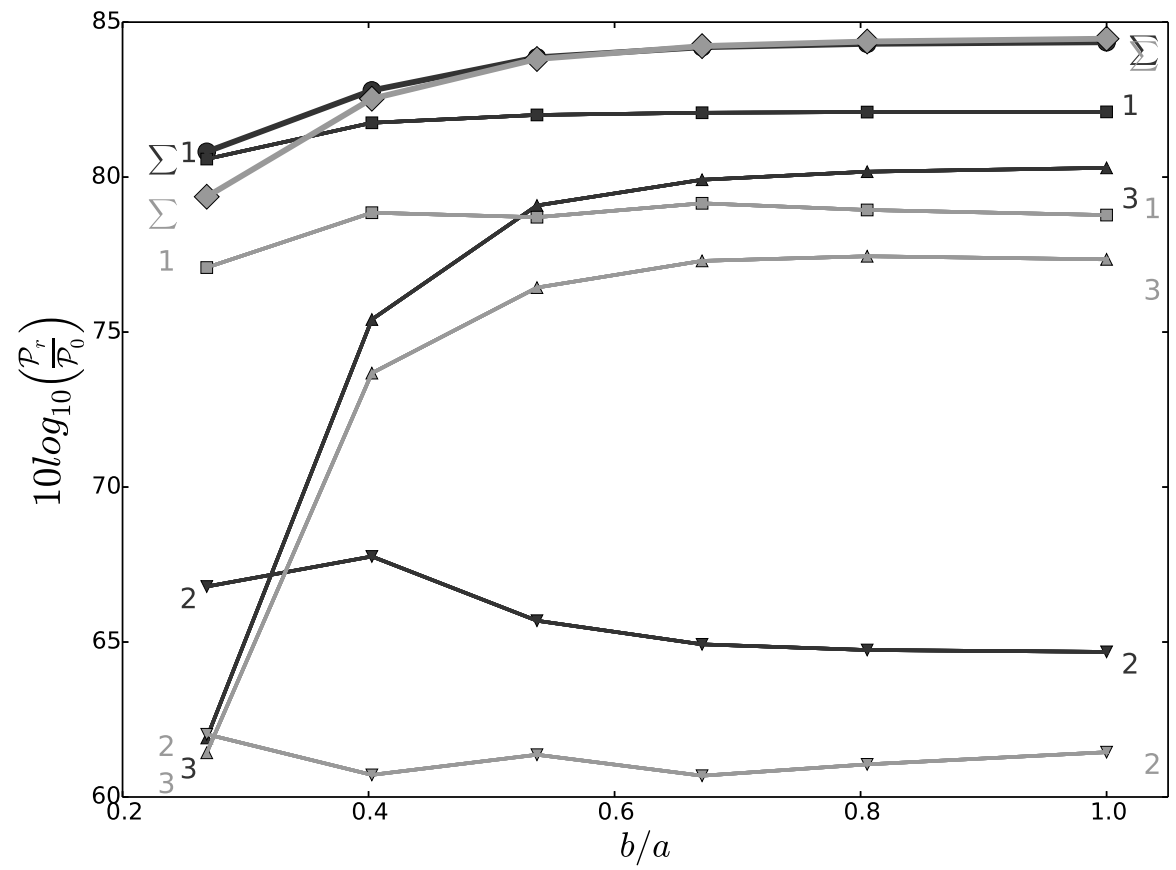

Figure 3: Comparison between analytical results (grey lines) and numerical results (black lines) for the three first harmonics and the total power. $\gamma=0.45 . \nabla$ Harmonic $2 ; \triangle$ Harmonic 3; $\square$ Harmonic 1; $\diamond$ Sum. 
total power, as shown in Fig. 3: the first harmonic is dominant.

\section{Numerical simulation}

With the help of the numerical tool developed by Guillemain et al. [14], a sound synthesis can be realized for both the non-beating and beating reed regimes. It is based again on the model by Wilson and Beavers, but takes the reed dynamics into account (as a single one-degree-of-freedom oscillator). Plausible values are $f_{r}=2341 \mathrm{~Hz}$ for the reed natural frequency and and $Q_{r}=1 / 0.8$ for the quality factor. The simulation uses the computed input impedance, from which a modal expansion is determined. The number of resonator modes is fixed to 4 , corresponding to contributions below approximately $3 \mathrm{kHz}$. The numerical solution is derived from that developed in [14], where each impedance mode is described as a second order IIR digital filter. In the discrete domain, the solving of the nonlinear characteristic equation remains analytical since the reed displacement discretization scheme leads to a one-sample delay between the reed displacement and the acoustic pressure ( $\mathrm{x}$ at sample $\mathrm{n}$ does not depend on $\mathrm{p}$ at sample $\mathrm{n}$ but at sample $\mathrm{n}-1$ ). This requires to solve simply a second order nonlinear equation instead of a multi-valued third order equation.

In order to reach the steady-state regime for different values of the excitation pressure $\gamma$, the computation implements a step function for $\gamma(t)$. The simulation provides the spectrum of the input pressure $p_{i n}$, then the transfer functions are calculated by using Eq. (8) (with a complex wavenumber). The difference of the model for the transfer admittance and that for the input impedance (a truncated modal expansion) is not important here because of the weakness of the higher harmonics. The number of harmonics considered here is only limited by the Nyquist frequency $(F e / 2=22050 \mathrm{~Hz})$. Obviously the model of the resonator is not suitable at very high frequencies, but the contribution of the highest harmonics to the radiated power is very small. For the tube with diaphragm, the playing frequency globally increases when the pressure $\gamma$ increases, about 30 cents between the oscillation and extinction thresholds. The increase is monotonous and slightly depends on the diaphragm radius. For narrow radii, the increase is much larger at low levels, because of inharmonicity. This frequency shift modifies the real part of the radiation impedance but has a negligible influence: a shift of 40 cents adds $0.2 \mathrm{~dB}$ on the radiated power. 


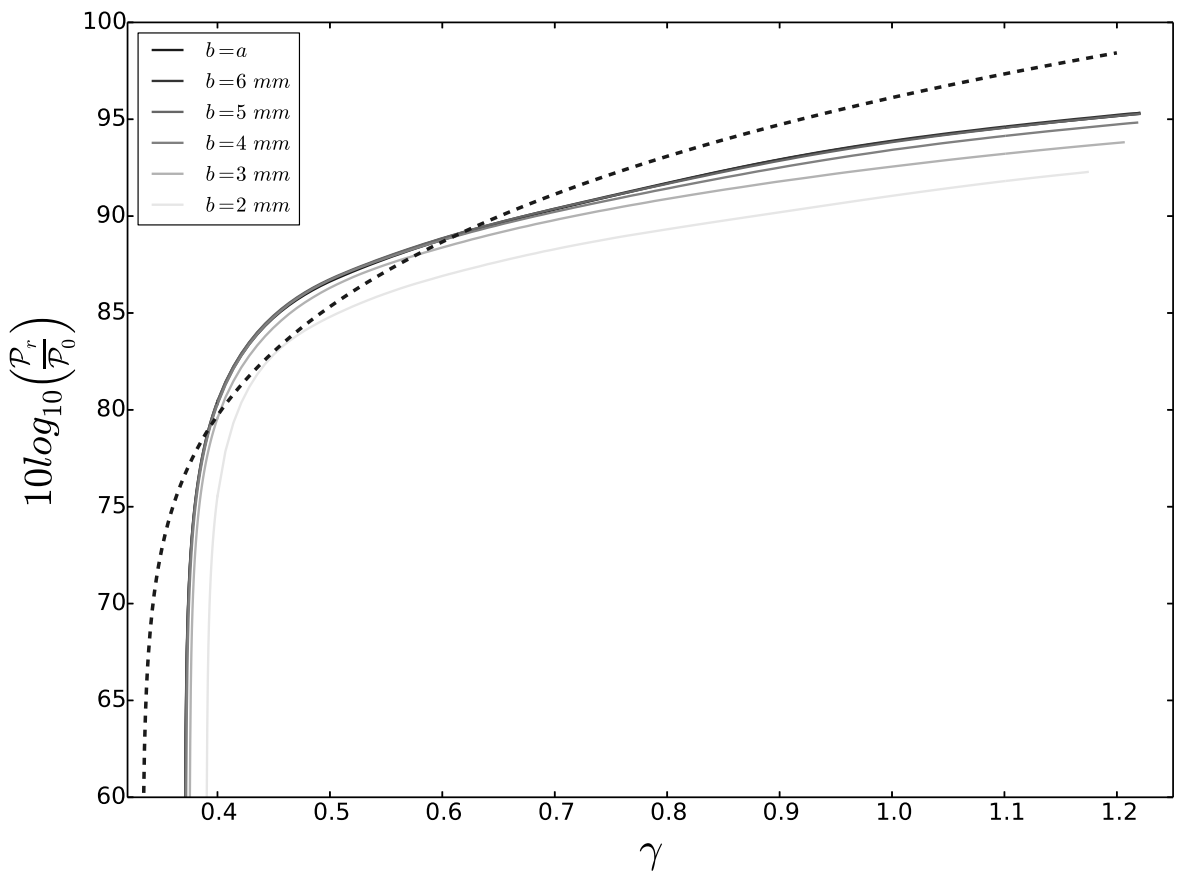

Figure 4: Radiated power for the six configurations, computed by simulation. The line shading is proportional to the diaphragm radius considered. Notice that the excitation pressure increases to high values, for which the reed beats The dotted line corresponds to the approximation of the simplest analytical model (Eq. (17)). 


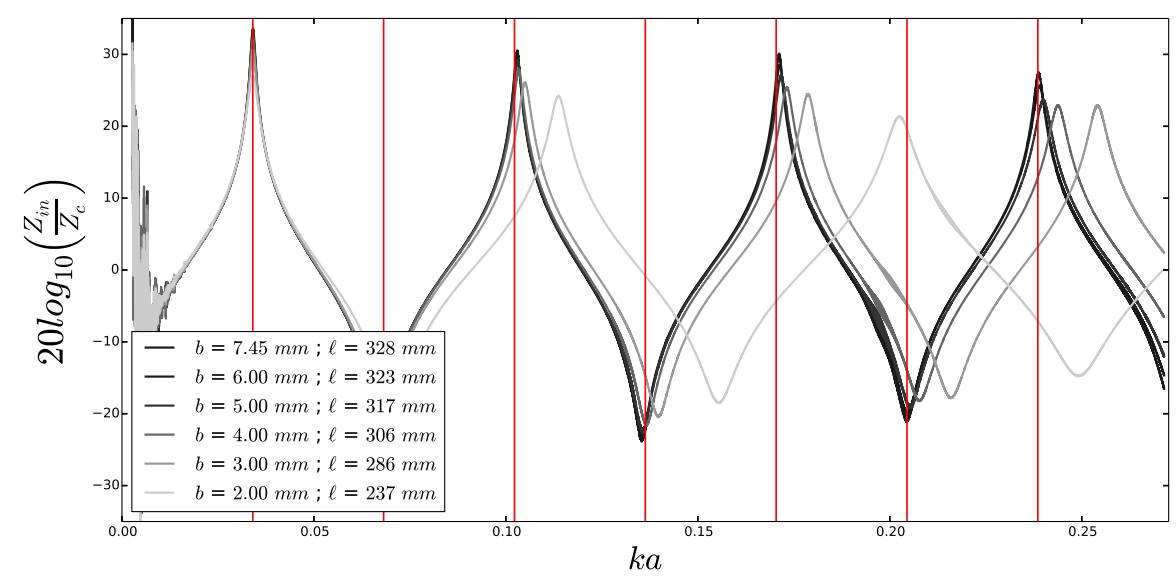

Figure 5: Input impedance for the different diaphragm radii (experimental result). The line shading is proportional to the diaphragm radius.

Fig. 3 shows a comparison of analytical and numerical results for the three first harmonics and the total power (limited to 3 harmonics for the analytical results), when $\gamma=0.45$. This figure shows that the radiated power does not depend on the opening size when the radius is sufficiently large. Also, the amplitude of the harmonics is over-estimated by the analytical formulas, but the two sums are nearly equal because the analytical result is not complete. Notice that because of inharmonicity the harmonic 2 becomes larger than the harmonic 3 for narrow diaphragms.

Fig. 4 shows the result for the different radii of diaphragms. In the beating reed regime and before extinction, the radiated power ratio between the 2 extreme configurations doesn't exceed $3 \mathrm{~dB}$ for a sound power level that approaches $95 \mathrm{~dB}$.

\section{Experiment}

\subsection{Input impedance measurement}

In order to evaluate the linear losses, the input impedance has been measured. The device used was built in Le Mans [15]. The pressure in the back cavity of the sound source is measured by a microphone, which gives an estimation of the flow rate. The source is a piezoelectric buzzer.

Six diaphragms were built in plastic (using a 3D printer device) and inserted in a wooden pipe, according to the details given in the introduction. 
Figure 5 shows the experimental results for the input impedance. As it was expected, the first resonance frequency is independent of the diaphragm radius. The mean deviation between resonance frequencies doesn't exceed the sensor precision evaluated to be 4 cents.

Experimental results were compared to theoretical results. For the pipe without diaphragm, the extrema of the impedance modulus are very well predicted by the calculation (the order of magnitude of the discrepancy is $1 \mathrm{~dB}$, equal to the precision of the sensor). However, the prediction of the extrema is less satisfactory for the pipe with diaphragm. For the case $b=2$ $\mathrm{mm}$, there is a discrepancy of $2.8 \mathrm{~dB}$ for the first peak, and the difference is slightly higher for the first dip and the second peak. To verify that this it not due to nonlinear dissipative phenomena, like vortex shedding, the pipe is excited at two different levels. This discrepancy is concluded to be the consequence of the underestimation of linear losses, which can be due to the edges of the diaphragm, or to the wall porosity and roughness (for the 3D printer used, the latter is $\left.R_{a}=6.4 \mu \mathrm{m}\right)$. Overall, the experiment shows a difference of $3.5+2.8 \mathrm{~dB}(3.5 \mathrm{~dB}$ is the theoretical result, see Section 3$)$ between the two extreme cases for the first impedance peak.

\subsection{External pressure measurement with an artificial mouth}

Experiments were carried out in order to compare the power emitted by all the tubes coupled to a composite reeds with a low stiffness and a mouthpiece. The radiated sound was measured with a microphone at a well defined distance away $(50 \mathrm{~cm})$, along the axis of the tubes. In order to ensure a good repeatability (during a measurement session) an artificial blowing machine was used. A study at realistic pressure levels was carried out for the instrument under playing conditions. Conversely, concerning reproducibility, a certain time variability of the set-up (latex lips - reed - mouthpiece coupling), is encountered. A similar in-vivo study on a musician would be less reproducible and also even less repeatable as the lip configuration changes constantly. The machine is a static-pressure controlled device provided with artificial lips [16]. The measurement of the static pressure in the downward cavity of the mouthpiece is performed with an Endevco piezoresistive pressure transducer $8507 \mathrm{C}-5$. Data acquisition was carried out at a $20 \mathrm{kHz}$ sampling frequency for a fixed 2-minute duration. Our interest is focused on the relative values between the different cases, not on a quantitative comparison between theory and experiment which implies a determination of the 
parameters of the mouthpiece and reed (and lips). This difficulty is somewhat overshadowed by the fact that the whole apparatus can be set in a position that allows running the self-oscillation on the first mode of the pipe only (i.e., not supported by other modes of the reed or the pipe). In this way, discrepancies concerning the radiated pressure do not exceed $1 \mathrm{~dB}$ on its stable part and $100 \mathrm{~Pa}$ for the thresholds (onset of the oscillation an extinction at high pressure) in a time interval corresponding to a measurement session. It was not possible to obtain a sound with the $2 \mathrm{~mm}$ diaphragm. For such a narrow diaphragm, the opening is comparable to the reed opening, a high steady flow velocity can be reached and strong convective sound absorption will occur [20]; moreover Wijnands and Hirschberg [21] have shown that for a single reed coupled to a tube with a narrow constriction, the nonlinear characteristic and consequently the extinction threshold can be strongly modified by the constriction.

The power is not measured in an anechoic room but during acoustic pressure measurements, the primary reflecting surfaces were covered with acoustic foam to minimize reflections. If the radiation is assumed to be that of a monopole, the pressure at a fixed distance from the orifice is a measure of the radiated power (the measurement was done in the axis of the tube). The external pressure sensor is a half-inch B\&K type 2669 linked to a nexus amplifier. The RMS sound pressure level is extracted with a $50 \mathrm{~ms}$ integration time. A slight change in the location of the microphone does not affect results, as expected.

The results are presented in two different graphs: for small and large excitation pressures, using a decreasing and an increasing linear pressure ramp, respectively. In order to obtain results for a wide range of excitation pressures, the force acting on the reed was changed between the measurements corresponding to the two experiments. For small blowing pressures (see Fig. 6 ), a weak slope of $3 \mathrm{kPa} /$ minute was chosen, and the acquisition was limited to $4 \mathrm{kPa}$. This allows the measurements to approach the oscillation threshold and reduces the phenomenon of bifurcation delay, as observed by Bergeot et al. for strong excitation-pressure slopes [17]. Conversely, if an increasing ramp was used, a jump at threshold would be observed. For the same reason only the decreasing ramps are represented in the figure.

Fig. 6 shows that for the two higher values of the diaphragm radius, $b=5$ and $6 \mathrm{~mm}$, the difference in radiated pressure with that of the pipe without diaphragm is less than $3 \mathrm{~dB}$. It is much more important for narrower diaphragms, around $15 \mathrm{~dB}$ between for $b=3 \mathrm{~mm}$. This difference is slightly 


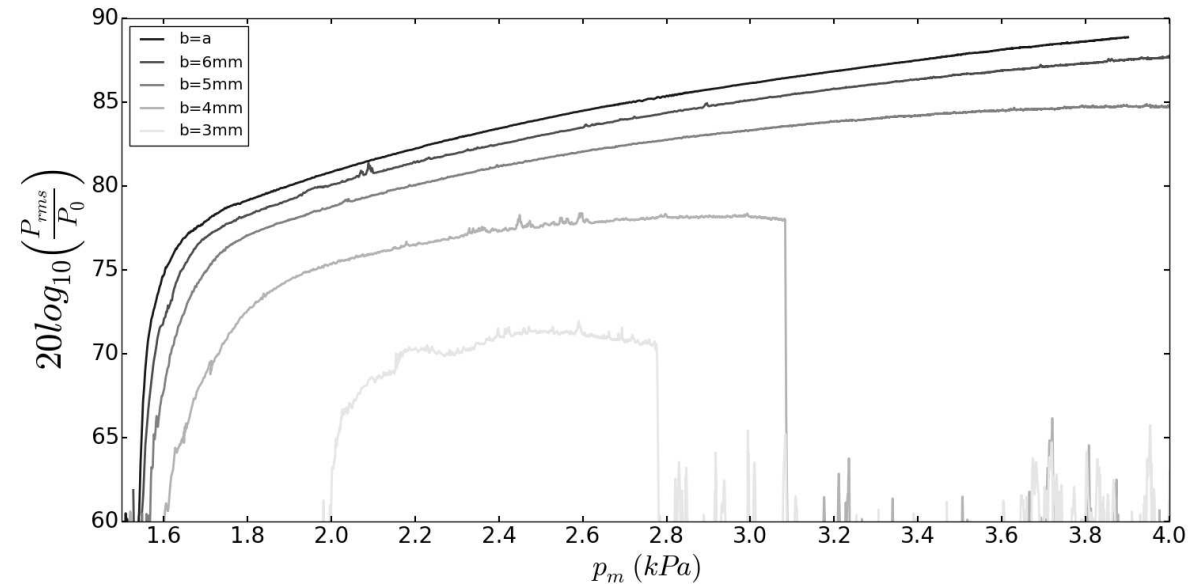

Figure 6: Measured sound pressure level radiated at a distance of $50 \mathrm{~cm}$ from the diaphragms. Sounds are produced by an artificial mouth with controlled static pressure. Only the results with a slowly decreasing pressure ramp $(3 \mathrm{kPa} /$ minute $)$ are represented in order to focus around the threshold region. The line shading is proportional to the diaphragm radius. No sound could be emitted with the $2 \mathrm{~mm}$ diaphragm.

larger than that found by calculation. Experiment confirms (Figs. 6 and 7) that the distance between the oscillation threshold, which tends to increase and the extinction one, which tends to decrease, is reduced when the orifice radius is decreased.

The cause of both features may lie in the underestimation of losses. Notice that losses can be either linear or nonlinear. In section 5.1, we remarked, for the measurement of the input impedance in a linear functioning, that linear losses in small holes are underestimated. However another phenomenon occurs: the nonlinear losses due to flow separation at the sharp edges of the orifice. This subject was investigated by Dalmont et al. for a tonehole [3] and for a bifurcation scheme of a cylindrical tube [9, 22].

\subsection{Experiments with diaphragms with round edges.}

In order to validate this assumption, we rounded the edges of the diaphragms. Figure 7 shows evidence for this effect on both the amplitude and the extinction threshold for two diaphragm radii. It is noticeable that for $b=5 \mathrm{~mm}$ the diaphragm with round edges radiates almost the same amplitude as when $b=a$. The curvature radius of the edges is $1.5 \mathrm{~mm}$, and both the internal and external sides are rounded. It has been checked that 


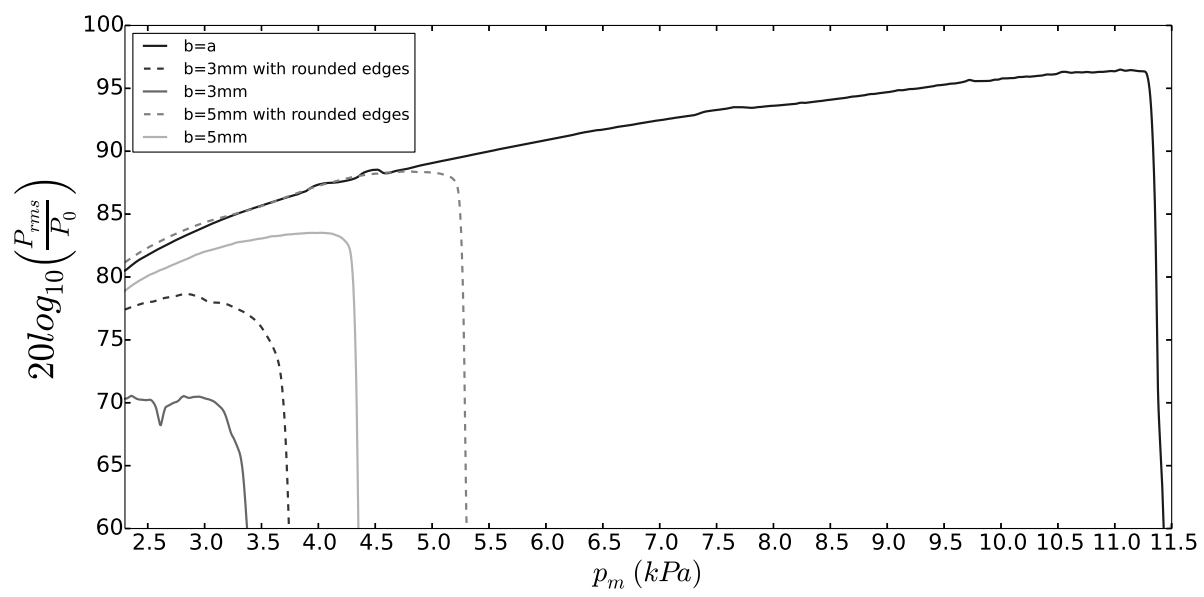

Figure 7: Measured sound pressure level radiated, for an increasing ramp, in conditions similar to Fig. 6, for diaphragms without and with rounded edges. Nonlinear losses are reduced by the round edges. In order to reach the highest extinction threshold for a pipe without diaphragm (just below $11.5 \mathrm{kPa}$ ) the increasing ramp of the excitation pressure is set to $11 \mathrm{kPa} / \mathrm{min}$. Notice that the abscissa range differs from that of Fig. 6. Solid lines: diaphragms with sharp edges. Dotted lines correspond to diaphragms with rounded edges.

with a smaller curvature radius $(0.7 \mathrm{~mm})$, the extinction threshold decrease to intermediate values. When applying a curvature on one side only, the nonlinear effects are larger than when applying on both sides. When applying a round edge on one side (either internal or external), the reduction of nonlinear losses seems to be independent of the side. However a curvature of $0.7 \mathrm{~mm}$ on both sides is more effective than a curvature of $\mathrm{r}=1.5 \mathrm{~mm}$ on one side.

\section{Conclusion}

The present work aimed to study the power radiated by reed woodwinds through holes of different diameters. The determination of the acoustic power based on a pressure measurement leads to interesting results for the comparison of the relative effect of the diaphragms. This does however not provide a quantitative comparison between the power measured and calculated. The use of cylindrical tubes with a reed-mouthpiece excitation allows considering a decreasing spectrum, with a dominant first harmonic. 
The following items can be concluded: i) For rather wide holes on cylindrical tubes, under the condition (11), the radiated power depends only weakly on the hole radius. The difference in radiated power remains much smaller than the difference obtained if the power ratio were proportional to the ratio in orifice cross-section areas; ii) The difference in power is mainly due to losses, either linear or nonlinear. If losses are taken into account, the condition (12) is not strict enough; iii) If nonlinear losses are reduced by rounding of the hole edges, the difference in power is largely reduced, and the extinction threshold significantly increases. As a consequence the distance between oscillation and extinction thresholds increases, and the dynamical range (between the onset of oscillation and the extinction pressure) is significantly increased; iv) Inharmonicity also plays a role in the total difference in power.

Qualitatively, the results might be extended to the case of some holes at the end of a cylindrical tube. However losses in side holes, especially when they are nonlinear, can have a different behavior, and can modify the sound production itself (e.g., the bifurcation curve). Moreover a supplementary difficulty occurs for a large number of holes. The interferences between different orifices as well as the existence of a cutoff frequency would require a measurement of the complete power radiated. It is known that a lattice of wide toneholes imply a high cutoff frequency [18, 19], but the effect of this cutoff frequency on radiation is not fully understood.

\section{Acknowledgements}

This work was financed by the research project CAGIMA supported by Agence Nationale de la Recherche. The authors thank A. Hirschberg, C. Vergez, W. Coyle and J.-P. Dalmont for fruitful discussions, and A. Busso, O. Pot et V. Long, as well as Buffet Group for the realization of the tubes and diaphragms.

\section{Appendix A. Model of the diaphragm}

Junction of the pipe and the diaphragm

The diaphragm has a very short thickness, $\ell_{d}$, of the same order of magnitude as that of the pipe wall, therefore it can be considered as a lumped element. Moreover, for the planar mode, the compliance, proportional to the volume, is very small and can be ignored. The acoustic mass of the planar mode inside the diaphragm is $M_{d}=\rho \ell_{d} / S_{d}$. The interaction of the evanescent modes inside the diaphragm [23] is assumed to be extremely weak, therefore 
on the left side, they act as a supplementary acoustic mass $M_{d i s c}$, while on the right side, the diaphragm radiates into infinite space as a semi-infinite pipe. The abrupt change in cross-section area was studied for a cylindrical duct [24] and the added mass was found to be:

$$
\begin{gathered}
M_{\text {disc }}=\frac{\rho}{b} \frac{4 \varepsilon^{2}}{\pi^{2}} K, \text { where } \\
K=-0.49198 \ln (\varepsilon)+0.50349 \\
-0.376246 \varepsilon^{2}-0.852222 \varepsilon^{2} \ln (\varepsilon)
\end{gathered}
$$

with $\varepsilon=1-b / a$. This formula is valid for $b / a>0.55$. The frequency dependence of the mass is ignored. A complementary expression is also available [24] for stronger discontinuities. Compared to $M_{d}$ and $M_{r}$, which are defined below $M_{d i s c}$ is small.

Radiation impedance of the diaphragm

The diaphragm wall presents a certain thickness corresponding to $a+w-b$ (see Fig. 1). This mainly affects the reactive part of the radiation impedance [25]. A fit formula for the imaginary part can be used for the intermediate cases, as follows:

$$
\begin{aligned}
\operatorname{Im}\left(Z_{r}\right) & =\omega M_{r}=\frac{\rho c}{S_{d}} k \delta_{d}, \text { where } \\
\delta_{d} & =\delta_{\infty}+\mu\left(\delta_{0}-\delta_{\infty}\right)+0.057 \mu\left[1-\mu^{5}\right] b
\end{aligned}
$$

$\mu=b /(a+w)$, and $\delta_{\infty}=0.8216 b$ and $\delta_{0}=0.6133 b$ correspond to the extreme cases, i.e. infinite flange and no flange, respectively, at low frequencies. Notice that $\delta_{\infty}$ and $\delta_{0}$ are frequency dependent [26]. For the low frequency approximation $(k b<<1)$, Eq.(A.2) is accurate enough. At higher frequencies wave reflections on the flange's edge have a noticeable influence (see [25], Eq.(42). Ignoring the frequency dependence, we can write the total value of the acoustic mass:

$$
M_{t}=M_{d}+M_{d i s c}+M_{r}
$$

Finally, the real part of $Z_{r}$ can be well approximated at low frequencies by the unflanged case Eq. (2).

[1] D. H. Keefe, Theory on the single woodwind tone hole, Journal of the Acoustical Society of America 72 (3) (1982) 676-687. 
[2] V. Dubos, J. Kergomard, A. Khettabi, J.-P. Dalmont, D.H. Keefe, C.J. Nederveen, Theory of sound propagation in a duct with a branched tube using modal decomposition, Acustica united with Acta Acustica 85 (2) (1999) 153-169.

[3] J.-P. Dalmont, C. J. Nederveen, V. Dubos, S. Ollivier, V. Meserette, E. te Sligte, Experimental determination of the equivalent circuit of an open side hole: linear and non linear behaviour, Acta Acustica united with acustica 88 (4) (2002) 567-575.

[4] A. Lefebvre, G. P. Scavone, Characterization of woodwind instrument toneholes with the finite element method, Journal of the Acoustical Society of America 131 (2012) 3153-3163.

[5] J.-P. Dalmont, C.J. Nederveen, Why is a saxophone louder than a clarinet, in: Proc. Institute of Acoustics, 195, 1997, pp. 267-272.

[6] A. Guilloteau, P. Guillemain, J. Kergomard, Dependence of the acoustic power produced by a woodwind on the tonehole size, in: International Symposium on Musical Acoustics, Le Mans, 2014, pp. 241-245.

[7] A.D. Pierce, Acoustics, Acoustical Society of America, 1989.

[8] T. A. Wilson, G. S. Beavers, Operating modes of the clarinet, Journal of the Acoustical Society of America 56 (1974) 653-658.

[9] J.-P. Dalmont, C. Frappé, Oscillation and extinction thresholds of the clarinet: Comparison of analytical results and experiments, Journal of the Acoustical Society of America 122 (2007) 1173-1179.

[10] P.-A. Taillard, J. Kergomard, F. Laloë, Iterated maps for clarinet-like systems, Nonlinear Dynamics (2010) 62: 253271.

[11] J. Kergomard, S. Ollivier, J. Gilbert, Calculation of the spectrum of selfsustained oscillators using a variable truncation method: Application to cylindrical reed instruments, Acustica united with Acta Acustica 86 (4) (2000) 685-703.

[12] N. Grand, J. Gilbert, F. Laloë, Oscillation threshold of woodwind instruments, Acustica 83 (1) (1997) 137-151. 
[13] D. H. Keefe, Acoustical wave propagation in cylindrical ducts: transmission line parameter approximations for isothermal and non-isothermal boundary conditions, Journal of the Acoustical Society of America 75 (1984) 58-62.

[14] P. Guillemain, J. Kergomard, T. Voinier, Real-time synthesis of clarinetlike instruments using digital impedance models, Journal of the Acoustical Society of America 118 (2005) 483-494.

[15] C.A. Macaluso, J.-P. Dalmont, Trumpet with near-perfect harmonicity: Design and acoustic results, Journal of the Acoustical Society of America 129 (2011) 404-414.

[16] D. Ferrand, C. Vergez, Blowing machine for wind musical instrument: toward a real-time control of the blowing pressure, in: 16th Mediterranean Conference on Control and Automation, IEEE, 2008, pp. 15621567.

[17] B. Bergeot, A. Almeida, C. Vergez, B. Gazengel, Prediction of the dynamic oscillation threshold in a clarinet model with a linearly increasing blowing pressure, Nonlinear Dynamics 73 (1-2) (2013) 521-534.

[18] A.H. Benade, S.N. Kouzoupis, The clarinet spectrum: Theory and experiment, Journal of the Acoustical Society of America 83 (1988) 292304.

[19] E. Moers, J. Kergomard, On the cutoff frequency of clarinet-like instruments. geometrical versus acoustical regularity, Acta Acustica united with Acustica 97 (2011) 984-996.

[20] P. Testud, Y. Aurégan, P. Moussou, A. Hirschberg, The whistling potentiality of an orifice in a confined flow using an energetic criterion, Journal of Sound and Vibration 325 (2009) 769780.

[21] A.P.J. Wijnands and A. Hirschberg, Effect of a pipe neck downstream a double reed, in Proceedings of Internation Symposium on Musical Acoustics, Dourdan (1995), 148-152.

[22] James M. Buick, M. Atig, D.J. Skulina, D.M. Campbell, J.-P. Dalmont, J.Gilbert, Investigation of non-linear acoustic losses at the open end of 
a tube, The Journal of the Acoustical Society of America, 129 (2011) 1261-1272.

[23] J. Kergomard, A. Garcia, G. Tagui, J.-P. Dalmont, Analysis of higher order mode effects in an expansion chamber using modal theory and equivalent electrical circuits, Journal of Sound and Vibration 129 (3) (1989) 457-475.

[24] J. Kergomard, A. Garcia, Simple discontinuities in acoustic waveguides at low frequencies: critical analysis and formulae, Journal of Sound and Vibration 114 (3) (1987) 465-479.

[25] J.-P. Dalmont, C.J. Nederveen, N. Joly, Radiation impedance of tubes with different flanges: Numerical and experimental investigations, Journal of Sound and Vibration 244 (3) (2001) 505-534.

[26] A.N. Norris, I.C. Sheng, Acoustic radiation from a circular pipe with an infinite flange, Journal of Sound and Vibration 135 (1) (1989) 85-93.

\section{List of Figures}

1 Geometry of the tube with diaphragm. The length $\ell$ depends on the diaphragm radius $b$, with a fixed first resonance frequency $f_{1}=250 \mathrm{~Hz}$. . . . . . . . . . . . . . . . 3

2 Calculated results from analytical formulas (??) and (??), for the 3 first harmonics below the beating reed regime $(\gamma=1 / 2)$. Dashed and plain lines correspond to the calculation with and without losses, respectively. The line shading is proportional to the diaphragm radius. Notice that losses produce both a shift of threshold and a decrease of the power. . . . . . . . . . 9

3 Comparison between analytical results (grey lines) and numerical results (black lines) for the three first harmonics and the total power. $\gamma=0.45 . \nabla$ Harmonic $2 ; \triangle$ Harmonic 3 ; Harmonic 1; $\diamond$ Sum. . . . . . . . . . . . . . . . . 10

4 Radiated power for the six configurations, computed by simulation. The line shading is proportional to the diaphragm radius considered. Notice that the excitation pressure increases to high values, for which the reed beats The dotted line corresponds to the approximation of the simplest analytical model (Eq. (??)). . . . . . . . . . . . . . . . . . 12 
$5 \quad$ Input impedance for the different diaphragm radii (experimental result). The line shading is proportional to the diaphragm radius. . . . . . . . . . . . . . . . . 13

6 Measured sound pressure level radiated at a distance of $50 \mathrm{~cm}$ from the diaphragms. Sounds are produced by an artificial mouth with controlled static pressure. Only the results with a slowly decreasing pressure ramp ( $3 \mathrm{kPa} /$ minute) are represented in order to focus around the threshold region. The line shading is proportional to the diaphragm radius. No sound could be emitted with the $2 \mathrm{~mm}$ diaphragm. . . . . . . . . . 16

7 Measured sound pressure level radiated, for an increasing ramp, in conditions similar to Fig. 6, for diaphragms without and with rounded edges. Nonlinear losses are reduced by the round edges. In order to reach the highest extinction threshold for a pipe without diaphragm (just below $11.5 \mathrm{kPa}$ ) the increasing ramp of the excitation pressure is set to $11 \mathrm{kPa} / \mathrm{min}$. Notice that the abscissa range differs from that of Fig. 6. Solid lines: diaphragms with sharp edges. Dotted lines correspond to diaphragms with rounded edges. . . . . . . . . . . . . . 17 